\title{
Collaborative remembering revisited: Study context access modulates collaborative inhibition and later benefits for individual memory
}

\author{
Magdalena Abel $^{1} \cdot$ Karl-Heinz T. Bäuml ${ }^{1}$
}

Published online: 2 August 2017

(C) Psychonomic Society, Inc. 2017

\begin{abstract}
Collaborating groups typically show reduced recall relative to nominal groups, i.e., to the cumulated nonredundant recall of the same number of people remembering in isolation-a finding termed collaborative inhibition. Motivated by the results of several previous studies, this study examined in two experiments whether access to study context at test influences the effects of collaboration. In both experiments, subjects collaborated in triads or recalled previously studied material in isolation. Experiment 1 applied short versus prolonged retention intervals to vary access to study context at test, whereas Experiment 2 used the list-method directed forgetting task and applied remember versus forget instructions to modulate context access. In both experiments, collaborative inhibition was present when access to study context at test was intact (i.e., after the short delay and the remember instruction) but was eliminated when the access was impaired (i.e., after the prolonged delay and the forget instruction). Also, post-collaborative gains for individual recall were greater when context access was impaired and collaborative inhibition was eliminated. The findings demonstrate a critical role of access to study context at test for collaborative inhibition, indicating that impaired context access may reflect a general boundary condition for the recall impairment. The possible role of context reactivation processes for beneficial effects of social recall is discussed.
\end{abstract}

Magdalena Abel

magdalena.abel@ur.de

1 Department of Experimental Psychology, Regensburg University, 93040 Regensburg, Germany
Keywords Memory retrieval - Social recall · Collaborative memory $\cdot$ Collaborative inhibition $\cdot$ Context reactivation

\section{Introduction}

Memory is typically conceptualized as lying entirely within an individual's mind. Consistently, the bigger part of research on human memory has examined memory of isolated individuals, studying and recalling various types of materials all by themselves. Such experiments have generated a wealth of knowledge on our memory system, but may nevertheless have neglected some important aspects of how we often use it in our daily lives. For instance, consider how often you remember and reminisce together with others. A variety of social settings may come to mind, ranging from family dinners to classroom or work discussions, pub meetings with friends, or even smalltalk with random strangers. Although this social aspect of memory is often ignored, many studies have by now investigated how memory is affected when we recall information together with others (e.g., Hirst \& Echterhoff, 2012; Rajaram \& PereiraPasarin, 2010; Weldon, 2000). Such research has uncovered many interesting aspects, but one of the most counterintuitive findings is probably that memory becomes worse when we engage in remembering together with others.

\section{Collaborative inhibition}

This issue is examined in studies on collaborative remembering. When memory of a collaborating group of several people is compared with individual memory, the collaborating group shows better recall than just a single individual (e.g., Hinsz, Tindale, \& Vollrath, 1997; Meudell, Hitch, \& 
Boyle, 1995). In the collaborative recall task, however, a more appropriate contrast is applied, and memory of a collaborating group is compared to memory of a so-called nominal group, a group in name only, which is formed by summing up the nonredundant responses of the same number of people remembering in isolation. Typically, in an initial study phase, subjects study materials (e.g., word lists) all by themselves and then, after a brief retention interval, attempt to recall the studied material, either in a collaborating group or individually. A comparison of collaborating and nominal groups typically shows that recall of collaborating groups is inferior to recall of nominal groups of individually working subjects, indicating that collaboration during recall may reduce the group's mnemonic potential (Basden, Basden, Bryner, \& Thomas, 1997; Weldon \& Bellinger, 1997; for a review, see Rajaram, 2011). The finding of reduced recall in collaborating compared to nominal groups is called collaborative inhibition. The effect has been shown to be very robust and to arise not only with word lists as study materials, but also with pictures, prose passages, emotional film, and spatial tasks (e.g., Sjolund, Erdman, \& Kelly, 2014; Weldon \& Bellinger, 1997; Wessel, Zandstra, Hengeveld, \& Moulds, 2015).

The most widely accepted account of collaborative inhibition states that collaborative remembering disrupts idiosyncratic retrieval strategies (see Basden et al., 1997). According to this account, after individual encoding, each group member brings their own and unique organization of the studied material to the task of collaborative remembering. During joint recall, the (differently organized) output of the other group members is assumed to collide with one's own retrieval strategy, disrupting its efficient use. Due to the disruption, on average, group members contribute less to collaborative recall, resulting in reduced performance compared to nominal groups without collaboration. Support for this explanation comes from studies showing that factors which strengthen and preserve stimulus organization also protect from collaborative inhibition. For instance, repeated encoding across several study and/or test cycles, or inducing similar ways of encoding in all group members, have been found to reduce or even eliminate collaborative inhibition (e.g., Basden et al., 1997; Congleton \& Rajaram, 2011; Finlay, Hitch, \& Meudell, 2000; Pereira-Pasarin \& Rajaram, 2011). However, newer proposals have also suggested the contribution of additional processes to the effect, such as retrieval inhibition of still to-berecalled information (e.g., Barber, Harris, \& Rajaram, 2015; for a recent meta-analysis, see Marion \& Thorley, 2016).

\section{Can collaboration be also beneficial for recall?}

A question still unresolved in research on collaborative remembering is whether collaboration can also be beneficial for memory. Subjective day-to-day experience suggests that this should be the case, and that input and cues from other persons can sometimes trigger memories. Imagine, for instance, you are attending a high school reunion, many years after graduation. Inevitably, you will be exposed to former classmates reminiscing about the past, which may then make you remember more and more from this time, memories you thought you had long forgotten. Such examples from subjective experience emphasize that there may indeed be conditions under which a social setting can facilitate remembering.

Previous research on collaborative remembering has devoted much effort to finding evidence for so-called effects of cross-cuing between collaborating subjects, i.e., instances in which one subject's response acts as an effective retrieval cue and prompts recall of further, otherwise not accessed information in another subject (Meudell, Hitch, \& Kirby, 1992; Meudell et al., 1995). However, to date, hardly any data support the existence of such cross-cuing effects. For instance, analyses of items lost and gained either during or after collaborative recall show that members of collaborating groups are not more likely than members of nominal groups to generate novel items that were not already recalled (Basden, Basden, \& Henry, 2000; Harris, Barnier, \& Sutton, 2013; Meudell et al., 1995; for an exception, see Meade, Nokes, \& Morrow, 2009, reporting cross-cuing in expert pilots).

At the very least, however, there is evidence that there may be a boundary condition to the negative effects of collaboration, namely a prolonged retention interval between study and test. Takahashi and Saito (2004) asked subjects to recall a story collaboratively or individually, and to do so either immediately after study (Experiment 1) or one week later (Experiment 2). Results showed collaborative inhibition when recall was tested after the short delay, but showed no such impairment after the longer delay, with recall of the collaborating groups being numerically, though not statistically, even higher than recall of the nominal groups. Congleton and Rajaram (2011) reported similar results, with intact collaborative inhibition on an immediate test, but eliminated collaborative inhibition after a 2-h delay. In parallel to Takahashi and Saito (2004), Congleton and Rajaram suggested that the disappearance of the recall impairment with delay could reflect processes of cross-cuing, which might become more effective when collaborating subjects rely less on their idiosyncratic retrieval strategies after delay.

\section{Beneficial effects in individual recall and in a different social setting}

Research on individual recall has predominantly documented negative influences of prior retrieval on recall of 
further information, as is reflected in studies on output interference (e.g., Roediger, 1974) and retrieval-induced forgetting (e.g., Anderson, Bjork, \& Bjork, 1994). Yet, more recent research suggests that retrieval does not always induce such detrimental effects, but can also cause beneficial effects on other memories. Indeed, across several studies, Bäuml and co-workers demonstrated that whereas retrieval typically impairs recall of other memories when access to study context during retrieval is intact, it can improve recall of other memories when the access is impaired. Access to study context in these studies was impaired by providing a forget cue after study (e.g., Bäuml \& Samenieh, 2010, 2012), engaging subjects in imagination tasks after study to change their internal context (e.g., Bäuml \& Samenieh, 2012), and increasing the length of the retention interval between study and test (e.g., Bäuml \& Schlichting, 2014). Prolonged retention intervals and imagination tasks induce contextual drift and thus, at test, create a mismatch between study and test context (e.g., Bower, 1972; Estes, 1955; Mensink \& Raaijmakers, 1988), which impairs study context access. Similarly, a forget cue after study may also change context (Sahakyan \& Kelley, 2002), or alternatively inhibit access to the whole study episode (Geiselman, Bjork, \& Fishman, 1983), thus again reducing access to the study context access (for a two-factor explanation of the two effects of retrieval in terms of inhibitory and context reactivation processes, see Bäuml \& Samenieh (2012), and General Discussion below; for a recent review of this research, see Bäuml, Aslan, \& Abel, 2017). ${ }^{1}$

Evidence that recall in a social setting may show similar retrieval dynamics as individual recall comes from a recent study by Abel and Bäuml (2015), who employed a variant of the speaker-listener task to study social recall. In the speaker-listener task, two subjects are tested together, and one of the two subjects is either asked to recall some target items first or listen to the other subject's preceding retrieval of the remaining (nontarget) items before trying to recall the target items herself (e.g., Cuc, Koppel, \& Hirst, 2007). Abel and Bäuml used this task but additionally manipulated access to study context at test by (i) providing a cue to either forget or remember previously studied items (Experiment 1), (ii) engaging subjects in an imagination task after study to create context change (Experiment 2), and (iii) varying the length of the retention interval between study and test ( 3 min versus 24 h; Experiment 3). Results showed that, when access to study context was intact at test, i.e., after the remember cue, in the absence of an imagination

\footnotetext{
${ }^{1}$ Similar to impaired access to study context, many other factors can also reduce recall levels, like impaired encoding or an increased interference level at test. Importantly, however, all of these factors can influence recall in very different ways, indicating that impaired context access is not just equivalent to reduced recall levels (for a demonstration, see Bäuml \& Samenieh, 2012).
}

task, and after the short retention interval, the listener's target recall was reduced by the speaker's preceding retrieval of the nontarget items. In contrast, when access to study context was impaired, i.e., after the forget cue, the imagination task, and the prolonged retention interval, the speaker's preceding retrieval improved the listener's target recall.

Whether access to study context at test can also affect collaborative recall has not been investigated to date, even though the collaborative recall task is the task predominantly used to assess recall in social groups. While the prior work by Abel and Bäuml (2015) suggests that social recall can show similar retrieval dynamics as individual recall, it is unclear whether, in a social setting, the finding is restricted to the well-controlled speaker-listener task or generalizes to the (less well-controlled) collaborativerecall task, in which three subjects interact in whichever way they prefer. In the speaker-listener task applied by Abel and Bäuml (2015) there is only one conversational turn and sequence of the two subjects' recall is fixed, with the subject acting as speaker starting recall and the subject acting as listener finishing the sequence after having been exposed to the speaker's prior recall. To achieve this experimental control, retrieval cues in the form of initial letters or even word stems are presented, and listeners are additionally required to monitor the speaker's performance for accuracy, ensuring that they are focusing on the task and retrieving along with the speaker (see also Cuc et al., 2007). In contrast, all these aspects of control are lacking from the collaborative recall task. In this task, usually three subjects are asked to engage in joint recall, but no restrictions with regard to sequence of recall are imposed upon subjects, no retrieval cues are provided, and whether subjects actually listen to each other in more than a superficial manner is unclear. Also, the recall task is usually more comprehensive and takes more time. Given all these differences between the two tasks, it is far from clear whether findings from the one task, i.e., the speaker-listener task, generalize to the other, i.e. the collaborative recall task, and vice versa.

The present study addresses the issue, examining whether beneficial effects of recall can also arise in the collaborativerecall task, at least if access to study context at test is sufficiently impaired. Two methods to impair study context access at test were employed in the present study that were also used in Abel and Bäuml's (2015) recent work: the presentation of a forget cue after study and a retention interval of $24 \mathrm{~h}$ between study and test. The aim was to investigate whether the results reported when using the speaker-listener task would generalize to collaborative recall, or whether, alternatively, beneficial effects of social recall are present in the speaker-listener task but do not arise with free collaboration. Importantly, the finding of a beneficial effect after a prolonged delay of $24 \mathrm{~h}$ would not necessarily be in conflict with the findings by Takahashi and Saito (2004) and Congleton and 
Rajaram (2011), who did not find (significant) beneficial effects after prolonged delay. Indeed, Takahashi and Saito (2004) reported at least a numerical trend for a beneficial effect after a 1-week delay, and Congleton and Rajaram (2011) did not observe a corresponding trend but used a delay interval of $2 \mathrm{~h}$ only, which may have been too short to create beneficial effects in collaborative recall.

\section{The present study}

The results of two experiments are reported designed to examine if access to study context at test influences effects of collaboration. Experiment 1 varied study context access by manipulating whether a short or a prolonged retention interval was placed before the final recall test. Experiment 2 varied study context access by providing either a forget cue or a remember cue after list study. In both experiments, subjects either collaborated in groups of three at test, or they were asked to engage in individual recall (and individual recall scores were used to compute recall scores of nominal groups). If results from the speaker-listener task generalize to the collaborative-recall task, then (i) detrimental effects of collaboration should arise when study context access is intact, i.e., after the short delay (Experiment 1) and after presentation of the remember cue (Experiment 2), and (ii) beneficial effects of collaboration should arise when study context is impaired, i.e., after the prolonged delay (Experiment 1) and after presentation of the forget cue (Experiment 2). In contrast, if the findings from the speakerlistener task do not generalize to collaborative recall-i.e., detrimental effects arise after short delay and the remember cue, but no beneficial effects arise in the remaining two conditions - this would indicate that different social settings can induce different retrieval dynamics.

While the results from the many previous studies on collaborative inhibition suggest that beneficial effects during collaboration may be hard to capture, there is evidence that benefits may arise more easily after collaboration, when subjects were asked to take another individual test after having engaged in a first round of collaborative or nominal recall. In fact, on such second tests, former members of collaborative groups typically show better recall compared to former members of nominal groups (e.g., Basden et al., 2000; Blumen \& Rajaram, 2008; Blumen \& Stern, 2011; Blumen, Young, \& Rajaram, 2014; Weldon \& Bellinger, 1997), and, likely, these post-collaborative gains arise due to reexposure. During collaboration, subjects may be exposed to output by their fellow group members that they would not have been able to recall themselves, so that collaborative remembering may be an opportunity for additional encoding (e.g., Barber \& Rajaram, 2011; Rajaram, 2011). While the primary goal of the present study was to investigate retrieval dynamics during collaboration, we also implemented individual tests (after collaborative versus nominal group recall) in the present study to more fully investigate the role of context access. On the basis of the hypothesis that study context access influences the consequences of collaboration during recall, we expected that it also influences the consequences of collaboration for later individual recall, with greater postcollaborative gains when context access is impaired and collaborative inhibition is absent (for prior findings on the issue, see Congleton \& Rajaram, 2011).

\section{Experiment 1}

Experiment 1 was conducted as a first test of the hypothesis that the consequences of collaborative remembering depend on study context access. We asked triads of subjects to study lists of unrelated words, try to recall them after a short or a prolonged retention interval, and do so either individually or in collaborating groups. On the basis of the literature on collaborative recall, we expected collaborative inhibition after the short retention interval, i.e., when access to the study context was still intact. In contrast, on the basis of the findings by Takahashi and Saito (2004), Congleton and Rajaram (2011), and Abel and Bäuml (2015), we expected no such recall impairment or even beneficial effects of collaboration after the prolonged retention interval, i.e., when access to the study context was impaired. After the first collaborative or nominal group test, subjects were moreover asked to complete a second individual memory test to assess whether post-collaborative gains depend on the presence of collaborative inhibition during collaboration. Following Congleton and Rajaram (2011), we expected greater post-collaborative gains in the absence than the presence of collaborative inhibition, i.e., greater gains after the prolonged than the short retention interval.

\section{Method}

Participants 180 students at Regensburg University were recruited for the experiment and received either course credit or 10 Euros for participation. Mean age was 22.3 years (range, 18-31 years). Sixty-seven subjects were male, 113 female. All subjects took part in the experiment in groups of three; whether group members knew each other before the onset of the experiment was not controlled (see Harris et al., 2013). Ninety subjects were asked to engage in collaborative recall, thus constituting 30 collaborating groups. The remaining 90 subjects were tested in triads as well, but were asked to work alone during recall; their individual recall scores were used to form the cumulative recall scores of 30 nominal groups. 
Material Item material comprised two item lists, each consisting of 30 nouns. Within lists, each noun was taken from a different semantic category (Van Overschelde, Rawson, \& Dunlosky, 2004; see Appendix A). Each of the two lists was equally often used as study material in the short and long delay conditions.

Design The experiment had a $2 \times 2$ mixed-factorial design. The first factor GROUP (collaborative, nominal) was manipulated between participants. Half of all subjects were asked to collaborate in groups of three and to work together when recalling the previously studied list. The other half of subjects were asked to work alone on all tests and their recall scores were used to compile nominal group recall. The second factor DELAY (5 min, $24 \mathrm{~h}$ ) was manipulated within participants. In the course of the experiment, all subjects were asked to study two item lists. Critically, one list was tested after a short delay of $5 \mathrm{~min}$, but the other was tested after a prolonged delay of $24 \mathrm{~h}$. Sequence of delay conditions was counterbalanced across subjects (for a similar procedure, see Abel \& Bäuml, 2015).

Procedure The experiment consisted of two blocks, each involving study and recall of one item list. On each experimental block, subjects were asked to try to memorize a list of words for an unspecified later test. The three subjects in each group always worked alone and on separate computers during encoding. Items were presented one at a time and for $4 \mathrm{~s}$ each centrally on the computer screen; order was set to random and was different for each subject. When list presentation was complete, subjects in the short delay condition worked on an unrelated cognitive test for $5 \mathrm{~min}$ before being asked to recall the list. In contrast, in the long delay condition, subjects worked on similar unrelated distractor tasks for $10 \mathrm{~min}$, but then were asked to leave the lab and to return after $24 \mathrm{~h}$ to complete the test.

At test, subjects in the collaborative groups were asked to work together during recall and try to remember the studied list as a group. The only instruction was that every group member should try to contribute to group recall as much as possible, but no regulations on how to collaborate exactly were imposed upon groups. Groups were given $3 \mathrm{~min}$ to collaborate and recall all list items; in addition to recording an audio file for this collaboration phase the experimenter also wrote down all responses that subjects generated on a sheet of paper. After $3 \mathrm{~min}$, subjects were given 1 additional min to go over the responses written down by the experimenter; they were allowed to cross out incorrect responses or to add new responses during this correction phase. In nominal groups, subjects were also given $3 \mathrm{~min}$ to recall the studied list items, but they were asked to work alone during remembering and to write their individual responses on a piece of paper. After $3 \mathrm{~min}$, in parallel to the collaborative groups, subjects in nominal groups were given 1 additional minute to go over their responses one more time. To foreshadow, results were in none of the present experiments affected by whether corrected recall scores after the additional minute were entered into the analyses or whether uncorrected recall scores after 3 min were used instead. Therefore, we will report corrected recall scores only when covering the results during collaborative vs. nominal group recall.

Because previous research has shown that former members of collaborating groups can show post-collaborative gains and enhanced memory compared to former members of nominal groups (e.g., Basden et al., 2000; Weldon \& Bellinger, 1997), a second recall test was conducted immediately after the first recall test. All subjects were asked to complete this second recall test individually. For subjects in collaborating groups, this was the first individual test on the item list; for subjects in nominal groups, it was a second individual test. Subjects were given $3 \mathrm{~min}$ and asked to write down all the list items they could remember, without any other specific instructions.

When the first experimental block was completed with the second recall test, subjects were asked to work on further unrelated distractor tasks for $5 \mathrm{~min}$. Afterwards, the second experimental block began with study of the second item list. The two experimental blocks were identical, the only exception being that recall of the studied list was tested after $5 \mathrm{~min}$ or $24 \mathrm{~h}$. Sequence of delay conditions was counterbalanced across participants and half of all subjects began the first experimental block with the short-delay condition, while the other half began with the long-delay condition. When the second experimental block was completed, subjects were debriefed and thanked for their participation.

\section{Results}

First Recall Test: Collaborative vs. Nominal Group Recall Fig. 1a shows mean recall as a function of GROUP (collaborative, nominal) and DELAY (5 min, $24 \mathrm{~h}$ ). A $2 \times 2$ ANOVA revealed no significant main effect of GROUP, $F(1,58)<1.0, p=.322, \eta^{2}=0.02$, but a significant main effect of DELAY, $F(1,58)=111.43, M S E=130.12$, $p<.001, \eta^{2}=0.66$, reflecting decreased recall after $24 \mathrm{~h}$ compared to $5 \min (61.1$ vs. $83.1 \%)$. Moreover, the ANOVA showed a significant interaction between DELAY and GROUP, $F(1,58)=10.89, M S E=130.12, p=.002$, $\eta^{2}=0.16$, suggesting that recall was differently affected by the group manipulation after shorter and longer delay. In fact, after the 5-min delay, collaboration at test impaired recall compared to the nominal group condition (78.0 vs. $88.2 \%), t(58)=2.80, p=.008, d=0.72$. After the 24$\mathrm{h}$ delay, however, no such collaborative inhibition emerged, and recall in collaborative groups was no longer different 

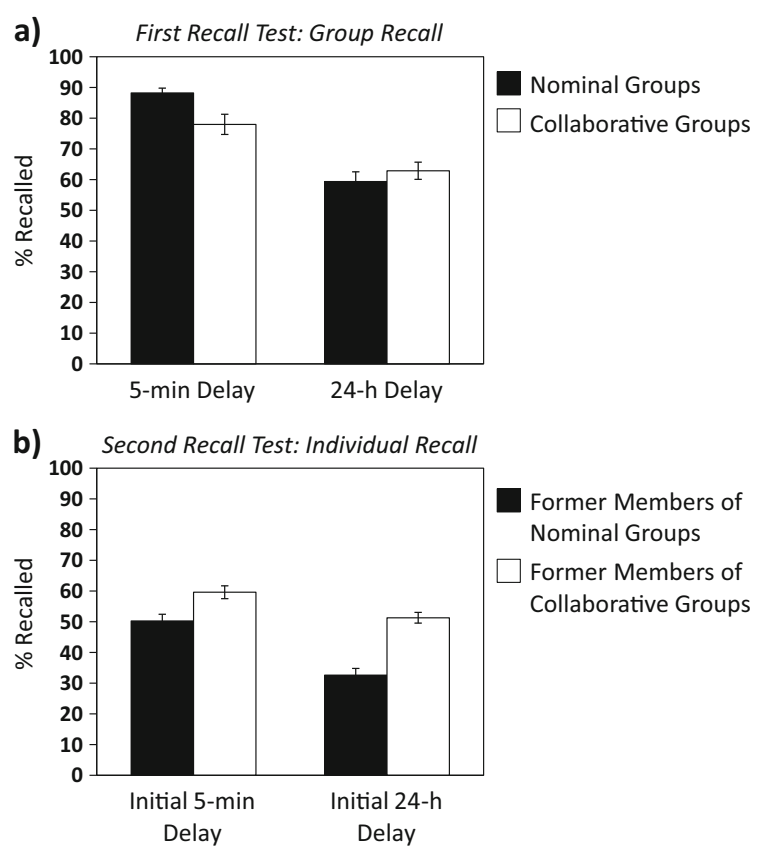

Fig. 1 Mean recall in Experiment 1. a Group recall on the first recall test (nominal vs. collaborative groups), conducted after $5 \mathrm{~min}$ or 24 h. b Individual recall on the second recall test (former members of nominal vs. collaborative groups), conducted right after the first recall test. Error bars represent \pm 1 standard errors

from nominal group recall, (62.9 vs. 59.4\%), $t(58)<1.0$, $p=.834, d=0.22$. Consistently, time-dependent forgetting from the 5-min to the 24-h delay was present in both types of groups, but was less pronounced in the collaborative group $(78.0$ vs. $62.9 \%), t(29)=5.64, p<.001$, $d=1.03$, than in the nominal group (88.2 vs. $59.4 \%)$, $t(29)=9.05, p<.001, d=1.65$.

Critically, the data in the long-delay conditions showed no difference between collaborative and nominal groups. Yet, null hypothesis significance testing cannot provide support for null hypotheses (see Gallistel, 2009; Wagenmakers, 2007), so we followed Masson's (2011) guidelines and used the Bayesian information criterion (BIC) to compute posterior probabilities for the null and alternative hypotheses being correct given the observed long-delay data (D). The resulting posterior probabilities were $\mathrm{P}_{B I C}\left(\mathrm{H}_{0} \mid \mathrm{D}\right)=0.844$ and $\mathrm{P}_{B I C}\left(\mathrm{H}_{1} \mid \mathrm{D}\right)=0.156$. Following Raftery (1995; see also Masson, 2011), this can be interpreted as positive evidence in favor of the null hypothesis.

Second Recall Test: Individual Memory after Collaborative vs. Nominal Group Recall Figure 1b shows mean individual recall as a function of prior GROUP affiliation during the first recall test (collaborative, nominal) and DELAY ( 5 min, 24 h). A $2 \times 2$ ANOVA showed a significant main effect of DELAY, $F(1,178)=103.98, M S E=$ $145.59, p<.001, \eta^{2}=0.37$, indicating that memory was reduced after the 24-h compared to the 5-min delay (42.0 vs. 54.9\%). This time, the ANOVA also showed a significant main effect of GROUP, $F(1,178)=28.84, M S E=$ $611.91, p<.001, \eta^{2}=0.89$, which reflects higher recall by former members of collaborative than nominal groups. However, the ANOVA also revealed a significant interaction between GROUP and DELAY, $F(1,178)=13.52$, $M S E=145.59, p<.001, \eta^{2}=0.07$, which suggests that the benefits of prior collaborative recall differed in size between the two delay conditions. Indeed, although recall of former members of collaborative groups was significantly enhanced in the 5-min delay condition (59.6 vs. $50.3 \%), t(178)=3.10, p=.002, d=0.46$, these postcollaborative gains were even more pronounced in the 24-h delay condition (51.3 vs. $32.6 \%), t(178)=6.71, p<.001$, $d=1.00$. Time-dependent forgetting arose irrespective of prior group affiliation, but individual recall followed the pattern observed for group recall. Forgetting from the 5-min to the 24-h delay was less pronounced in individual recall of former collaborative group members (59.6 vs. 51.3\%), $t(89)=5.22, p<.001, d=0.55$, compared to recall of former nominal group members $(50.3$ vs. $32.6 \%), t(89)=$ $8.88, p<.001, d=0.94$.

\section{Discussion}

The results of Experiment 1 show the typical detrimental effect of collaboration after short delay, with reduced recall in collaborative relative to nominal groups. In contrast, the results show an elimination of the effect after prolonged delay, with the two types of groups showing roughly similar recall levels. These results indicate a critical role of delay for the effects of collaboration, which is also reflected in later individual recall. Indeed, post-collaborative gains were present after short and prolonged delay, but were more pronounced after prolonged delay and in the absence of collaborative inhibition. These results mimic those reported by Takahashi and Saito (2004) and Congleton and Rajaram (2011), generalizing them to the present experimental conditions. In particular, the findings are consistent with the view of a critical role of study context access for the effects of collaboration. If this is the case, then the results of Experiment 1 should generalize to other situations in which access to study context is impaired at test, like, for instance, list-method directed forgetting. Experiment 2 examined the issue.

\section{Experiment 2}

Experiment 2 employed the list-method directed forgetting task to examine the effects of collaboration on recall when 
access to the study context at test is impaired. In this task, subjects study two lists of items and, between study of the two lists, are cued to either remember the first list for a subsequent test or forget the list, pretending that it was presented just for practice or by mistake (Bjork, 1970, 1989). At test, subjects are then asked to recall list 1 , irrespective of original cuing. The typical finding is that the forget cue reduces recall of list 1 items relative to the remember cue (e.g., Geiselman et al., 1983). The forgetting of list 1 items is often explained by impaired access to the list- 1 study context, be it by inhibitory or noninhibitory mechanisms (e.g., Geiselman et al., 1983; Pastötter \& Bäuml, 2010; Sahakyan \& Kelley, 2002). On the basis of such explanations, we expected the typical detrimental effect of collaborative recall in the remember condition, i.e., when access to the study context was still intact, but expected no such recall impairment, or even beneficial effects of collaboration, in the forget condition, i.e., when access to the study context was impaired. Similarly, we expected larger post-collaborative gains in the forget than in the remember condition. Such pattern of results would strengthen the proposal that access to study context can play a critical role for the effects of collaborative recall.

\section{Method}

Participants 192 students took part in the experiment in return for either course credit or 10 Euros. Mean age was 23.0 years (range, 18-30 years). Fifty-one subjects were male, 141 female. 96 participants collaborated in groups of three, providing the data of 32 collaborating groups; whether group members knew each other before the onset of the experiment was not controlled. The remaining 96 subjects were also tested in triads but completed individual memory tests, providing the data of 32 nominal groups.

Material Two sets of item material were compiled. Each set comprised two lists, with each list containing 24 items. Items on each list were again taken from different semantic categories (Van Overschelde et al., 2004; see Appendix A). Material sets were equally often used in remember and forget conditions; additionally, sequence of lists in each set was counterbalanced across participants.

Design The experiment had a $2 \times 2$ mixed-factorial design. The first factor GROUP (collaborative, nominal) was again manipulated between participants, in parallel to Experiment 1 . The second factor CUE (remember, forget) was manipulated within participants. In the course of the experiment, all subjects were asked to complete two blocks of the same task, on each block successively studying two item lists. Critically, when study of the first list was complete, subjects received a remember cue for this list on one of the two blocks and were asked to try to keep on remembering it for a later test. On the other experimental block, they were instead cued to try to forget the first list, pretending that it would not be tested later (see Bjork, 1970; Geiselman et al., 1983). Sequence of cue conditions was counterbalanced across subjects.

Procedure The experiment consisted of two blocks, each involving study and recall of two lists of items. On each experimental block, subjects were asked to try to memorize two lists of words. During study phases, the three subjects in each group worked alone and on separate computers. List items were presented one at a time and for $4 \mathrm{~s}$ each centrally on the computer screen. Order of items was set to random and was different for each subject. After list-1 study, subjects received a remember cue for the list on one of the two blocks, but a forget cue on the other block. When the remember cue was provided, subjects were asked to remember the just studied list for a later test, and to additionally memorize the second list for the later test. In contrast, when the forget cue was provided, subjects were instead informed that the studied list was for practice only and would not be tested later. Subjects were asked to try to forget the list and to memorize the next list instead, which would indeed be tested later. Members of the same group always received the same cues and sequence of cue conditions was counterbalanced across groups (for prior work showing that sequence of cue conditions in within-subjects designs does not affect the directed forgetting effect, see Bäuml \& Samenieh, 2012; Conway \& Fthenaki, 2003; Zellner \& Bäuml, 2006). After cue presentation, subjects were asked to study the second item list. List 2 was then presented in parallel to list 1.

On each experimental block, subjects were asked to work on an unrelated (geographic) distractor task for $3 \mathrm{~min}$ before being tested. For group recall, two separate tests were conducted for list 1 and list 2, and because we were primarily interested in group memory for list 1 after forget compared to remember cues, list 1 was always tested before list 2 . The test procedure was identical to that applied in Experiment 1. During initial group tests, subjects had 3 min for recall (plus $1 \mathrm{~min}$ for corrections). During subsequent individual recall tests, all subjects were asked to work alone and given $3 \mathrm{~min}$ per list to write down all the items that they could remember. Prior work shows that testing list 1 before list 2 affects list2 recall, making it hard to interpret list-2 results (Golding \& Gottlob, 2005; Pastötter \& Bäuml, 2010; Pastötter, Kliegl, $\&$ Bäuml, 2012). For this reason, we will focus exclusively on list-1 recall in the results section of the main text. For reasons of completeness, we provide an analysis of list- 2 recall in Appendix B. 
Between experimental blocks, subjects were asked to work on further unrelated distractor tasks for $5 \mathrm{~min}$. Afterwards, the second experimental block began and subjects were again asked to study two (new) item lists. The two experimental blocks were identical, the only difference being whether a forget or remember cue for list 1 was presented between presentation of the two lists. If a forget cue had been presented on the first block, participants were ensured that no second (false) forget cue would be presented on the second block (see Zellner \& Bäuml, 2006). When both experimental blocks had been completed, subjects were debriefed and thanked for their participation.

\section{Results}

First Recall Test: Collaborative vs. Nominal Group Recall Figure 2a shows mean group recall of list 1 on the first conducted test as a function of GROUP (collaborative, nominal) and CUE (remember, forget). A $2 \times 2$ ANOVA showed neither a significant main effect of GROUP on recall, $F(1,62)=3.44, p=.068, \eta^{2}=0.05$, nor a significant main effect of CUE, $F(1,62)=1.53, M S E=203.91$, $p=.220, \eta^{2}=0.02$. There was, however, a significant interaction between CUE and GROUP, $F(1,62)=4.92$, $M S E=203.91, p=.030, \eta^{2}=0.07$, suggesting that the consequences of collaborative remembering depended on
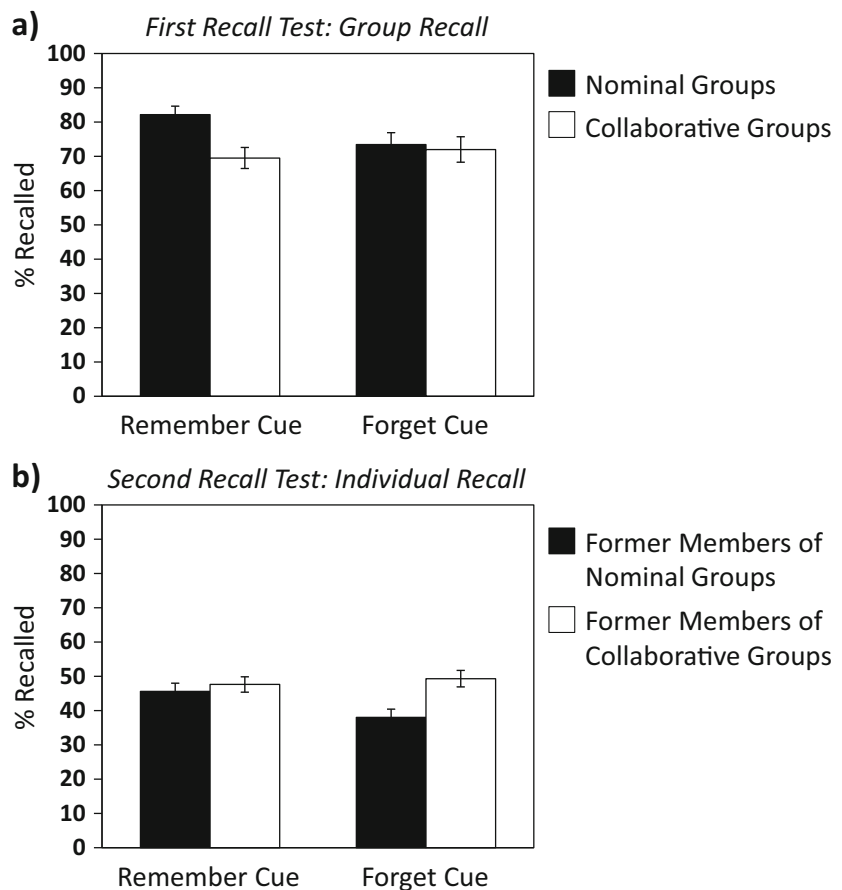

Fig. 2 Mean list-1 recall in Experiment 2. a Group recall on the first recall test (nominal vs. collaborative groups). b Individual recall on the second recall test (former members of nominal vs. collaborative groups). Error bars represent \pm 1 standard errors
CUE. After the remember cue, collaboration at test impaired recall compared to the nominal group condition (69.5 vs. $82.2 \%), t(62)=3.19, p=.002, d=0.80$. In contrast, after the forget cue, no collaborative inhibition was found and recall in collaborative groups did not differ from nominal group recall (72.0 vs. $73.4 \%), t(62)<1.0, p=.779$, $d=0.07$. Forget compared to remember cues impaired recall only for nominal groups (73.4 vs. 82.2\%), $t(31)=$ 2.38, $p=.024, d=0.42$, but not for collaborative groups (72.0 vs. $69.5 \%), t(31)<1.0, p=.482, d=0.13 .^{2}$

Critically, the data in forget conditions showed no difference between collaborative and nominal groups. We again used the Bayesian information criterion (BIC) to compute posterior probabilities for the null and alternative hypotheses being correct given the observed data (D) in the forget conditions. The resulting posterior probabilities were $\mathrm{P}_{B I C}\left(\mathrm{H}_{0} \mid \mathrm{D}\right)=0.885$ and $\mathrm{P}_{B I C}\left(\mathrm{H}_{1} \mid \mathrm{D}\right)=0.115$. Following (Raftery, 1995); see also Masson (2011), this can be interpreted as positive evidence in favor of the null hypothesis.

\section{Second Recall Test: Individual Memory after Collabo-} rative vs. Nominal Group Recall Figure 2b shows mean individual recall of list 1 as a function of prior GROUP affiliation during the first test (collaborative, nominal) and CUE (remember, forget). A $2 \times 2$ ANOVA showed no significant main effect of CUE, $F(1,190)=3.33, M S E=251.52$, $p=.070, \eta^{2}=0.02$, but revealed a significant main effect of GROUP, $F(1,190)=5.21, M S E=813.01, p=$ $.024, \eta^{2}=0.03$, which was accompanied by a significant interaction between GROUP and CUE, $F(1,190)=8.23$, $M S E=251.52, p=.005, \eta^{2}=0.04$. In the remembercue condition, individual memory was not enhanced after collaborative compared to nominal group recall (47.6 vs. $45.6 \%), t(190)<1.0, p=.541, d=0.09$. In the forget-cue condition, however, we found a significant benefit of prior collaboration compared to nominal group recall for subsequent individual performance (49.3 vs. $38.0 \%$ ), $t(190)=3.32, p=.001, d=0.48$. Interestingly, in former members of nominal groups, directed forgetting persisted from group recall to individual recall, with lower recall after forget compared to remember cues (38.0 vs. $45.6 \%)$, $t(95)=3.19, p=.002, d=0.33$. Former members of collaborative groups did not show directed forgetting (49.3 vs. $47.6 \%), t(95)<1.0, p=.441, d=0.08$, a pattern that corresponds to the group recall phase.

\footnotetext{
${ }^{2}$ Intact list-method directed forgetting was not only present in nominal group recall, but was also present when mean individual recall of nominal group members was analyzed and compared across forget and remember cues (38.8 vs. $46.3 \%), t(95)=3.23, p=.002, d=0.33$.
} 


\section{Discussion}

The results show the typical detrimental effect of collaboration in the remember condition, with reduced recall in the collaborative compared to the nominal groups. In contrast, the results show no such effect in the forget condition, with both types of groups showing roughly equivalent recall rates. Consistently, there was also an effect of collaboration on final individual recall, showing stronger benefits of collaboration in the forget than in the remember condition. The results in the remember condition thus parallel those in the short-delay condition of Experiment 1, and the results in the forget condition parallel those in the long-delay condition. Together, these findings are consistent with the view that access to the study context at test modulates the effects of collaboration on recall.

In Experiment 1, a collaborative inhibition effect arose after the short delay, but no longer after the longer delay. In consequence, amount of time-dependent forgetting was reduced in collaborative recall relative to nominal recall. Similarly, in Experiment 2, a collaborative inhibition effect arose in response to the remember cue, but no longer in response to the forget cue. Because amount of forgetting induced by the forget cue was small relative to the timedependent forgetting observed in Experiment 1, in Experiment 2 the directed forgetting effect was present in nominal recall, but did not survive group recall. Likely, if a forget cue induced more forgetting than it did in the present study (for an example, see Bäuml \& Kliegl, 2013, Experiments 1A and $1 \mathrm{~B}$ ), directed forgetting, like time-dependent forgetting, would be reduced in collaborative recall, but still be present. Future work may address this prediction.

\section{Additional exploratory analyses}

Audio files recorded during collaboration in Experiments 1 and 2 were additionally reanalyzed and coded for several interaction variables, like number of conversational turns or instances of cross-cuing (see Harris, Keil, Sutton, Barnier, \& McIlwain, 2011 for details; see Appendix C for descriptive statistics on all coded variables). ${ }^{3}$ Most importantly, only the variables reflecting cross-cuing showed consistent patterns across experiments. Mean number of instances in which subjects successfully cued each other to recall previously unmentioned information was affected by delay in Experiment 1 and type of cue in Experiment 2. Although rare overall, successful cuing more frequently occurred after $24 \mathrm{~h}$ than 5 min (means: .41 vs. .15), $F(1,26)=5.14$,

\footnotetext{
${ }^{3}$ Due to technical and/or experimenter error, the necessary audiofiles for these exploratory reanalyses were only available for 27 of the 30 collaborating groups tested in Experiment 1, and for 26 of the 32 collaborating groups tested in Experiment 2.
}

$M S E=.18, p=.032, \eta^{2}=0.17$, and more frequently occurred after the forget than after the remember cue $(.35 \mathrm{vs}$. $.04), F(1,25)=8.16, M S E=.15, p=.008, \eta^{2}=0.25$. Mean number of failed cuing attempts was also higher after longer than shorter delay (.96 vs. .44), $F(1,26)=4.87$, $M S E=.75, p=.036, \eta^{2}=0.16$, and, at least numerically, after the forget than after the remember cue (.27 vs. $.15), F(1,25)=1.86, M S E=.09, p=.185, \eta^{2}=0.07$. Thus, when study context access was impaired (after longer delay and the forget cue), subjects more frequently and more successfully cross-cued each other. This holds while numbers of cross-cuing attempts were generally close to floor levels, which may limit conclusions to some extent.

As another exploratory analysis, we additionally coded how much each group member contributed to the group task (see Appendix $\mathrm{C}$ for details). On average, the group member that contributed the most delivered $45.1 \%$ of all group nominations, followed by $32.6 \%$ from the next group member and $22.3 \%$ from the weakest group member. The distribution of contributions across group members did not differ between short and long delay conditions in Experiments 1 or between remember and forget cue conditions in Experiment 2, all $\chi^{2} \mathrm{~s}<$ $1.0, p s \geq .892$. Yet, group members who contributed the most did not always stay the same across these within-subjects conditions; in fact, in $58.5 \%$ of the analyzed collaborating groups the subject that contributed the most changed (i.e., across delay or cue conditions). Nevertheless, this additional analysis could indicate that blocking or even social loafing may have played a role in the present experiments.

\section{General discussion}

The primary goal of the present study was to investigate if the consequences of collaborative remembering depend on access to study context at test. Whereas in Experiment 1, access to study context was manipulated by varying the delay interval between study and test, in Experiment 2, access was manipulated by presenting a remember cue versus a forget cue after study. Consistent with prior work (Congleton and Rajaram, 2011; Takahashi \& Saito, 2004), Experiment 1 showed that collaborative remembering is detrimental after a short retention interval, but leads to recall rates comparable to those of a nominal control group after prolonged retention interval. An analogous pattern was observed in Experiment 2, with collaborative remembering resulting in collaborative inhibition when a remember cue was provided after study, but to no longer impair recall when a forget cue was provided. These findings indicate that the consequences of collaborative remembering depend upon whether, at test, access to the study context is intact or impaired. When context access is largely intact (after short delay or remember instructions), collaboration induces 
inhibition; when context access is impaired (after longer delay and forget instructions), no such inhibition arises.

\section{Boundary conditions of collaborative inhibition}

The present results are most parsimoniously explained by the assumption that retrieval disruption or inhibition operate when access to study context is intact, but that such processes are negligible, or even nonexistent, when context access is impaired. Such situation may arise if subjects no longer rely as heavily on their idiosyncratic retrieval strategies when context access is impaired, or if the interference level of the items is reduced under such conditions, so that inhibition no longer operates. This one-factor account of the present results suggests that the present long delay and forget conditions reflect boundary conditions of collaborative inhibition.

Congleton and Rajaram (2011) already suggested that delay may serve as a boundary condition of collaborative inhibition. Solely based on the finding that delay eliminates collaborative inhibition, one could argue that memories may simply consolidate across prolonged delay (Müller \& Pilzecker, 1900; McGaugh, 2000), thus making them less susceptible to negative effects of collaboration (e.g., retrieval-strategy disruption). The present results, however, also show that a forget instruction after study can serve as a boundary condition of collaborative inhibition. Because a forget cue is not known to trigger consolidation processes, the observed elimination of collaborative inhibition in this case should not be mediated by consolidation processes. Rather, on the basis of the present results and the above one-factor explanation of the results, the prediction arises that all factors that impair study context access at test may serve as boundary conditions of collaborative inhibition. In fact, access to study context can not only be impaired by increasing the retention interval between study and test or by providing a forget cue after study, but, for instance, may also be induced if imagination or semantic generation tasks follow item encoding (i.e., Divis \& Benjamin, 2014; Jang \& Huber, 2008; Pastötter \& Bäuml, 2007; Sahakyan \& Kelley, 2002).

Also retrieval practice has been argued to drive internal context change (e.g., Criss \& Shiffrin, 2004; Jang \& Huber, 2008; Jonker, Seli, \& MacLeod, 2013). If so, retrieval practice may also impair study context access and thus, like a prolonged retention interval or a forget cue, serve as a boundary condition of collaborative inhibition. Results by Congleton and Rajaram (2011) are indeed consistent with such view. In their study, Congleton and Rajaram examined the role of prior testing for collaborative inhibition and found that repeated individual retrieval practice prior to collaboration can eliminate collaborative inhibition. However, because the exact mechanisms mediating retrieval practice effects are still a matter of dispute, further work is required to examine in more detail why retrieval practice can reduce collaborative inhibition.

\section{Collaborative-recall "versus" speaker-listener task}

The present study found no evidence for a reversal of collaborative inhibition, and thus, no evidence for cross-cuing, which fits with prior reports in the literature on collaborative recall (e.g., Basden et al., 2000; Meudell et al., 1992, 1995). Even though our exploratory analyses showed that collaborating groups more frequently and more successfully attempted to cross-cue each other when context access was impaired and recall was harder, such instances were rare overall and not enough to enhance recall above the nominal group recall baseline and thus show benefits of collaboration. Abel and Bäuml (2015) recently addressed the issue of possible benefits of social recall employing the speaker-listener task. Although the present study employing the collaborative recall task used two methods to impair access to study context-i.e., increasing the retention interval between study and test and providing a forget cue after study - that were also employed in Abel and Bäuml (2015), the present results turned out to be only partly consistent with the results from the prior work. Indeed, whereas both studies found detrimental effects of social recall when access to study context was intact, when access to study context was impaired beneficial effects of social recall arose in the speaker-listener task only.

Abel and Bäuml (2015) explained their findings by means of a two-factor account, which assumes that, in general, recall of a speaker triggers two types of processes in the listener: inhibition and blocking of interfering memories and context reactivation processes. The proposal then was that primarily inhibition and blocking may operate in a listener's recall when access to study context is intact, whereas primarily context reactivation processes may be induced when the access is impaired (see also Bäuml \& Samenieh, 2012). When access to study context is impaired, retrieval of single items by the speaker may reactivate the study context in the listener (e.g., Greene, 1989; Thios \& D'Agostino, 1976), which may then serve as a retrieval cue for the listener's recall of the remaining items and thus improve recall performance (Howard \& Kahana, 2002; Raaijmakers \& Shiffrin, 1981). The present results are consistent with such a two-factor account as well. Indeed, recall of one person of a group may trigger two types of memory processes in the group's other persons: retrieval disruption and inhibition on the one hand and context reactivation on the other. Critically, the relative contribution of the two types of processes may depend on study context access, and retrieval disruption and inhibition play a more dominant role when 
context access is maintained, and context reactivation play the more dominant role when context access is impaired. This two-factor account can explain the present finding of collaborative inhibition when access to study context is intact and a reduction, or even elimination, of the effect when the access is impaired.

Depending on whether the present findings are explained by means of the one-factor or the two-factor account, the results suggest quantitative or qualitative differences between the collaborative-recall and the speaker-listener task. When following the more parsimonious one-factor account, the results indicate that context reactivation plays a role in the speakerlistener task but not in the collaborative-recall task. In contrast, when following the two-factor account, the results suggest that the two tasks differ in amount of context reactivation only, with context reactivation playing a more important role in the speaker-listener than the collaborative-recall task. Obviously, to distinguish between the two accounts, it is important to figure out what exactly causes the difference in results between the two social recall tasks.

One factor contributing to the difference in results between studies may be that the speaker-listener task creates tight experimental control on individuals' roles during social recall, whereas subjects interact freely in the collaborativerecall task. This relative lack of experimental control in the collaborative-recall task may influence the results. For instance, during collaboration, some (more extravert) subjects may take over the task, whereas other (less extravert) subjects may hold back, thus reducing overall recall. In fact, our exploratory analyses show that, on average, recall was in all conditions steered by one rather dominant subject, contributing the most to all group nominations. Potentially, such unbalanced contributions across group members may reduce recall of the whole group and do so particularly when recall is hard, like after a prolonged retention interval or in response to a forget instruction. Similarly, social loafing may have played a role for the present results. Because only group (and not individual) scores are recorded during collaboration, each single subject may feel less accountable and contribute less to group performance. Prior work has shown that this is not what causes collaborative inhibition after short delays (see Weldon et al., 2000), but social loafing may become more important when recall gets hard, like, for instance, when access to study context is impaired.

Discovering exactly what factors create the difference in results between the two tasks might become an important task for future research on social recall. The results from such work may also provide an answer on whether context reactivation processes operate in the speaker-listener task only or are also present in the collaborative-recall task, thus providing a clue on whether social recall differs quantitatively or qualitatively between tasks. In general, research of the past decades illustrates how hard it is to detect benefits and cross-cuing effects during collaborative recall (see Rajaram \& Maswood, in press, for a recent discussion). The results of the present study fit well within this picture.

\section{Beneficial effects of collaboration in individual recall}

In the present study, context access did not only influence effects of collaboration during group recall, but did also affect recall on a subsequent individual recall test. The present findings are consistent with prior work by Congleton and Rajaram (2011), which reported beneficial effects of preceding collaboration that were larger after longer delay than after short delay, also when retrieval practice in the form of studytest cycles was applied during encoding. Consequently, Congleton and Rajaram (2011) concluded that postcollaborative gains can be larger under conditions that eliminate collaborative inhibition. The present findings replicate this pattern for prolonged delay and extend it to list-method directed forgetting. Post-collaborative gains likely reflect reexposure effects, with subjects in collaborating groups being reexposed to a number of items that were recalled by their fellow group members, but that they would not have been able to recall themselves (Barber \& Rajaram, 2011; Basden et al., 2000; Rajaram, 2011). Following this reasoning, the present findings may indicate that subjects were reexposed to a larger number of such items during collaboration when context access was impaired than when it was maintained.

Potentially, the difference in post-collaborative gains could reflect an effect of context reactivation: When context access is impaired, context reactivation processes may create a larger number of items recalled by each single subject that were not recallable by the other subjects, thus creating the potential for stronger reexposure effects. The present as well as the prior findings by Congleton and Rajaram (2011) are generally consistent with such a proposal. Yet, at least for now, it remains unclear if context reactivation per se or the ensuing elimination of collaborative inhibition is the critical ingredient for the higher postcollaborative recall gains observed in the present and the prior work. Future work may examine this issue in greater detail.

\section{Conclusions}

In sum, the present findings demonstrate that access to study context at test plays a critical role for social recall. Its exact role may depend upon the type of social setting, but with an unrestricted collaborative-recall task impaired access to study context may at the very least act as a boundary condition to collaborative inhibition, which generalizes and extends prior work on the issue. Because context 
access affects collaborative inhibition, it also influences subsequent individual remembering and post-collaborative gains. Remembering together with others is not always detrimental to recall, but can become more beneficial when access to the study episode is impaired and context reactivation is a precondition for successful recall.

Author Note We thank J. Binapfl, V. Haller, T. Krauss, C. Nottberg, E. Rose, S. Rosskopf and L. Wallner for their help with data collection.

\section{Appendix A}

In the following, we provide all item lists used in the present experiments. Since the study was conducted in German, we additionally provide German translations in parentheses.

\section{Item material used in Experiment 1}

List A: mushroom, box, bell, belt, dog, chemistry, grandma, stool, plane, spoon, blackbird, shoulder, tangerine, flute, lightning, letter, Italy, desert, copper, pepper, radio, moth, butter, policeman, screw, ring, spade, fir, silk, vinegar [Pilz, Kiste, Glocke, Gürtel, Hund, Chemie, Oma, Hocker, Flugzeug, Löffel, Amsel, Schulter, Mandarine, Flöte, Blitz, Brief, Italien, Wüste, Kupfer, Pfeffer, Radio, Motte, Butter, Polizist, Schraube, Ring, Spaten, Tanne, Seide, Essig]

List B: phone, plate, drum, chair, wine, heart, storm, tie, carpenter, hill, cabbage, tent, golf, water, maple, roof, tango, worm, bus, uncle, gun, apple, second, wool, cinnamon, church, puzzle, ferry, bucket, power [Telefon, Teller, Trommel, Sessel, Wein, Herz, Sturm, Krawatte, Schreiner, Hügel, Kohl, Zelt, Golf, Wasser, Ahorn, Dach, Tango, Wurm, Bus, Onkel, Gewehr, Apfel, Sekunde, Wolle, Zimt, Kirche, Rätsel, Fähre, Eimer, Strom]

\section{Item material used in Experiment 2}

List A: pants, fork, finger, door, donkey, tulip, football, hail, sword, chair, dragonfly, perfume, wine, building, tomato, uncle, mint, meter, ferry, year, letter, bronze, plow, train [Hose, Gabel, Finger, Tür, Esel, Tulpe, Fußball, Hagel, Schwert, Sessel, Libelle, Parfüm, Wein, Gebäude, Tomate, Onkel, Minze, Meter, Fähre, Jahr, Brief, Bronze, Pflug, Zug]

List B: tongue, vinegar, blouse, rat, snow, pan, pistol, hotel, hammer, cotton, stream, piano, shelf, pencil, bike, fir, alarm, cucumber, shovel, cloud, earrings, newspaper, doll, bone [Zunge, Essig, Bluse, Ratte, Schnee, Pfanne, Pistole, Hotel, Hammer, Watte, Bach, Klavier, Regal,
Stift, Fahrrad, Tanne, Wecker, Gurke, Schaufel, Wolke, Ohrring, Zeitung, Puppe, Knochen]

List C: knife, paper, phone, shoe, nose, lightning, desert, lamp, lilac, pliers, chicken, cabbage, coin, whistle, tent, lawyer, flea, tennis, story, cloth, wall, liquor, bus, cinnamon [Messer, Papier, Telefon, Schuh, Nase, Blitz, Wüste, Lampe, Flieder, Zange, Huhn, Kohl, Münze, Pfeife, Zelt, Anwalt, Floh, Tennis, Geschichte, Stoff, Mauer, Likör, Bus, Zimt]

List D: lake, nail, violin, cup, sun, pig, ring, radio, palm, mountain, monastery, second, golf, apricot, worm, milk, office, niece, stick, vulture, sage, power, sofa, bean [See, Nagel, Geige, Becher, Sonne, Schwein, Ring, Radio, Palme, Berg, Kloster, Sekunde, Golf, Aprikose, Wurm, Milch, Büro, Nichte, Stock, Geier, Salbei, Strom, Sofa, Bohne]

\section{Appendix B}

\section{Analysis of List-2 recall in Experiment 2}

Instructions to forget a previously studied list in the listmethod directed forgetting task does typically not only cause reduced memory for list 1 , but also enhanced memory for list 2 (e.g., Bjork, 1970). This list-2 enhancement has been suggested to arise because the forget cue reduces interference of list 1 (e.g., Geiselman et al., 1983). Critically, however, several prior studies indicate that the benefit for list-2 recall can be reduced and even eliminated when list 1 is tested before list 2 (e.g., Golding \& Gottlob, 2005; Pastötter \& Bäuml, 2010; Pastötter et al., 2012). Testing list 1 first may reinstate the list's interference potential, which decreases the benefits for list- 2 recall (see Pastötter et al., 2012) and, consequently, makes list- 2 data harder to interpret. Our focus in Experiment 2 was clearly on list-1 recall, so that recall of list 1 was always tested first. For reasons of completeness, we will nevertheless report the results for list-2 recall below.

First Recall Test: Collaborative vs. Nominal Group Recall Concerning group recall of list 2, a $2 \times 2$ ANOVA revealed a significant main effect of CUE, $F(1,62)=4.07$, $M S E=208.00, p=.048, \eta^{2}=0.06$, but no significant main effect of GROUP, $F(1,62)=1.03, M S E=460.12$, $p=.315, \eta^{2}=0.02$, and also no significant interaction between CUE and GROUP, $F(1,62)<1.0, M S E=$ 208.00, $p=.526, \eta^{2}=0.01$. Thus, collapsing across group conditions, list- 2 recall was higher after forget than remember cues ( 74.2 vs. $69.0 \%$ ). Yet, this difference did not remain significant when differentiating between collaborative groups (73.1 vs. $66.3 \%), t(31)=1.72, p=.095, d=$ 0.31 , and nominal groups ( 75.3 vs. $71.7 \%), t(31)=1.08$, $p=.287, d=0.19$. 
Second Recall Test: Individual Memory after Collaborative vs. Nominal Group Recall Concerning individual recall of list 2, a $2 \times 2$ ANOVA showed a significant main effect of CUE, $F(1,190)=9.50, M S E=196.20$, $p=.002, \eta^{2}=0.05$, a significant main effect of GROUP, $F(1,190)=20.47, M S E=670.64, p<.001, \eta^{2}=$ 0.10 , and a significant interaction between GROUP and CUE, $F(1,190)=5.40, M S E=196.20, p=.021$, $\eta^{2}=0.03$. Individual memory was enhanced after collaborative compared to nominal group recall (50.2 vs. $38.2 \%$ ), and these post-collaborative gains were stronger after forget cues (54.0 vs. $38.8 \%), t(190)=5.27, p<.001, d=0.76$, than after remember cues $(46.3$ vs. $37.7 \%), t(190)=2.78$, $p=.006, d=0.40$. Moreover, list-2 recall was higher after forget compared to remember cues (46.4 vs. $42.0 \%$ ), but this difference was only significant for former members of collaborative groups (54.0 vs. $46.3 \%), t(95)=3.68, p<.001$, $d=0.33$. not for former members of nominal groups ( 38.8 vs. $37.7 \%), t(95)<1.0, p=.577, d=0.08$.

\section{Appendix C}

\section{Additionally coded variables: interaction during collaboration}

We followed the coding scheme reported by Harris et al. (2011) and coded number of conversational turns as well as how frequently acknowledgments, repetitions, corrections, elaborations as well as successful and failed cuing attempts were part of the collaboration phase. Harris et al. additionally coded strategy disagreements and references of task expertise; however, such exchanges never occurred in the present experiments. Table 1 summarizes means and standard deviations for all coded interaction variables, separately for Experiments 1 and 2; in addition, the table also details what proportion of conversational turns the single variables amounted to.

We only reported analyses on cross-cuing variables in the main text because these were most important for the pursued research question and were consistent across experiments. However, Table 1 shows means for all coded interaction variables and illustrates that other variables may show differences across experiments. For instance, subjects in Experiment 1 spent a greater proportion of conversational turns with acknowledging contributions by other group members and elaborating on their own recall than subjects in Experiment 2, all $F s(1,51) \geq 4.82, M S E \leq .004$, $p \leq .033, \eta^{2} \geq 0.09$, whereas subjects in Experiment 2 corrected each other more often than subjects in Experiment $1, F(1,62)=5.29, M S E=.005, p=.026$, $\eta^{2}=0.09$. These differences may relate to procedural differences between experiments, with subjects in Experiment 1 studying one item list before being tested, and subjects in Experiment 2 studying two lists, thus likely suffering from interference. Yet, since we did not manipulate

Table 1 Mean frequencies for all coded interaction variables as well as mean proportion of conversational turns, separately for conditions in Experiments 1 and 2

\begin{tabular}{|c|c|c|c|c|c|c|c|c|}
\hline \multirow{6}{*}{ Experiment 1} & \multirow{4}{*}{5 -min delay } & \multicolumn{7}{|c|}{ Mean frequencies } \\
\hline & & Turns & Acknowledgments & Repetitions & Corrections & Elaborations & Successful cues & Failed cues \\
\hline & & 39.00 & 4.15 & 4.96 & 3.04 & 1.26 & .15 & .44 \\
\hline & & (13.04) & $(2.32)$ & $(4.08)$ & $(2.19)$ & $(1.38)$ & $(.36)$ & (.93) \\
\hline & 24-h delay & 31.56 & 3.67 & 3.30 & 2.04 & 1.85 & .41 & .96 \\
\hline & & (13.08) & $(2.40)$ & $(5.24)$ & $(1.70)$ & $(2.11)$ & $(.57)$ & $(1.02)$ \\
\hline \multirow[t]{6}{*}{ Experiment 2} & Remember cue & 35.00 & 2.50 & 3.00 & 3.69 & 1.04 & .04 & .15 \\
\hline & & $(14.20)$ & $(1.86)$ & $(2.71)$ & $(2.62)$ & $(1.93)$ & $(.20)$ & $(.37)$ \\
\hline & Forget cue & 33.46 & 2.89 & 4.46 & 3.73 & .92 & .35 & .27 \\
\hline & & $(12.02)$ & $(1.86)$ & $(4.58)$ & $(3.05)$ & $(1.44)$ & $(.49)$ & $(.45)$ \\
\hline & & & \multicolumn{6}{|c|}{ Mean proportions of turns } \\
\hline & & & Acknowledgments & Repetitions & Corrections & Elaborations & Successful cues & Failed cues \\
\hline \multirow[t]{4}{*}{ Experiment 1} & 5-min delay & & .11 & .15 & .08 & .03 & .004 & .01 \\
\hline & & & (.04) & (.19) & $(.05)$ & (.03) & $(.01)$ & $(.02)$ \\
\hline & 24-h delay & & .12 & .09 & .07 & .06 & .01 & .03 \\
\hline & & & (.08) & (.13) & (.07) & $(.06)$ & $(.02)$ & $(.04)$ \\
\hline \multirow[t]{4}{*}{ Experiment 2} & Remember cue & & .07 & .08 & .10 & .02 & .002 & .003 \\
\hline & & & $(.05)$ & $(.07)$ & (.06) & (.04) & $(.01)$ & $(.01)$ \\
\hline & Forget cue & & .09 & .12 & .11 & .02 & .01 & .01 \\
\hline & & & $(.06)$ & (.10) & $(.08)$ & (.04) & $(.01)$ & $(.02)$ \\
\hline
\end{tabular}

Values in parentheses represent standard deviations 
Table 2 Mean number of group nominations as well as proportion of group nominations by subject (ranked for proportion contributed within groups), separately for conditions in Experiments 1 and 2

\begin{tabular}{|c|c|c|c|c|c|}
\hline & & \multirow[t]{2}{*}{$\begin{array}{l}\text { Mean number of } \\
\text { group nominations }\end{array}$} & \multicolumn{3}{|l|}{$\begin{array}{l}\text { Proportions of group } \\
\text { nominations }\end{array}$} \\
\hline & & & Subjects with rank 1 & Subjects with rank 2 & Subjects with rank 3 \\
\hline \multirow[t]{4}{*}{ Experiment 1} & 5-min delay & 27.63 & .43 & .33 & .25 \\
\hline & & $(6.78)$ & $(.07)$ & $(.05)$ & $(.05)$ \\
\hline & 24-h delay & 21.33 & .47 & .31 & .22 \\
\hline & & $(5.95)$ & $(.11)$ & $(.07)$ & $(.08)$ \\
\hline \multirow[t]{4}{*}{ Experiment 2} & Remember cue & 23.04 & .44 & .33 & .23 \\
\hline & & $(5.23)$ & $(.06)$ & $(.05)$ & $(.07)$ \\
\hline & Forget cue & 21.81 & .47 & .34 & .20 \\
\hline & & $(6.46)$ & (.07) & $(.05)$ & $(.07)$ \\
\hline
\end{tabular}

Values in parentheses represent standard deviations

interference in a targeted and controlled fashion, the value of such comparisons across experiments may be limited.

\section{Single group members' contributions to the group task}

As described in the main text we also coded how many nominations each single group member contributed to the group recall task. For each triad, we ranked group members according to their contributed number of nominations, so the subject with rank 1 showed the highest, the subject with rank 2 the second highest, and the subject with rank 3 the lowest number of nominations. We then calculated what proportion of all group nominations the single subjects' contributions corresponded to. Table 2 summarizes these data separately for all conditions in Experiments 1 and 2.

\section{References}

Abel, M., \& Bäuml, K.-H. T. (2015). Selective memory retrieval in social groups: When silence is golden and when it is not. Cognition, 140, 40-48.

Anderson, M. C., Bjork, R. A., \& Bjork, E. L. (1994). Remembering can cause forgetting: Retrieval dynamics in long-term memory. Journal of Experimental Psychology: Learning, Memory, and Cognition, 20, 1063-1087.

Bäuml, K.-H. T., Aslan, A., \& Abel, M. (2017). The two faces of selective memory retrieval - cognitive, developmental, and social processes. In Ross, B. H. (Ed.), Psychology of learning and motivation, (Vol. 66 pp. 167-209). Elsevier Inc.: Academic Press.

Bäuml, K.-H. T., \& Kliegl, O. (2013). The critical role of retrieval processes in release from proactive interference. Journal of Memory and Language, 68, 39-53.

Bäuml, K.-H. T., \& Samenieh, A. (2010). The two faces of memory retrieval. Psychological Science, 21, 793-795.

Bäuml, K.-H. T., \& Samenieh, A. (2012). Selective memory retrieval can impair and improve retrieval of other memories. Journal of
Experimental Psychology: Learning, Memory, \& Cognition, 38, 488-494.

Bäuml, K.-H. T., \& Schlichting, A. (2014). Memory retrieval as a selfpropagating process. Cognition, 132, 16-21.

Barber, S. J., Harris, C. B., \& Rajaram, S. (2015). Why two heads apart are better than two heads together: Multiple mechanisms underlie the collaborative inhibition effect in memory. Journal of Experimental Psychology: Learning, Memory, and Cognition, 41, 559-566.

Barber, S. J., \& Rajaram, S. (2011). Collaborative memory and part-set cuing impairments: The role of executive depletion in modulating retrieval disruption. Memory, 19, 378-397.

Basden, B. H., Basden, D. R., Bryner, S., \& Thomas, R. L. (1997). A comparison of group and individual remembering: Does collaboration disrupt retrieval strategies? Journal of Experimental Psychology: Learning, Memory, and Cognition, 23, 11761189.

Basden, B. H., Basden, D. R., \& Henry, S. (2000). Costs and benefits of collaborative remembering. Applied Cognitive Psychology, 14, 497-507.

Bjork, R. A. (1970). Positive forgetting: The noninterference of items intentionally forgotten. Journal of Verbal Learning and Verbal Behavior, 9, 255-268.

Bjork, R. A. (1989). Retrieval inhibition as an adaptive mechanism in human memory. In Roediger, H. L., \& Craik, F. I. M. (Eds.), Varieties of memory and consciousness: Essays in honour of Endel Tulving (pp. 309-330). Hillsdale, NJ: Erlbaum.

Blumen, H. M., \& Rajaram, S. (2008). Influence of re-exposure and retrieval disruption during group collaboration on later individual recall. Memory, 16, 231-244.

Blumen, H. M., \& Stern, Y. (2011). Short-term and long-term collaboration benefits on individual recall in younger and older adults. Memory \& Cognition, 39, 147-154.

Blumen, H. M., Young, K. E., \& Rajaram, S. (2014). Optimizing group collaboration to improve later retention. Journal of Applied Research in Memory and Cognition, 3, 244-251.

Bower, G. H. (1972). Stimulus-sampling theory of encoding variability. In Melton, A. W., \& Martin, E. (Eds.), Coding processes in human memory (pp. 85-121). New York: Wiley.

Congleton, A. R., \& Rajaram, S. (2011). The influence of learning methods on collaboration: Prior repeated retrieval enhances retrieval organization, abolishes collaborative inhibition, and promotes post-collaborative memory. Journal of Experimental Psychology: General, 140, 535-551. 
Conway, M. A., \& Fthenaki, A. (2003). Disruption of inhibitory control of memory following lesions to the frontal and temporal lobes. Cortex, 39, 667-686.

Criss, A. H., \& Shiffrin, R. M. (2004). Context noise and item noise jointly determine recognition memory: A comment on Dennis and Humphreys (2001). Psychological Review, 111, 800-807.

Cuc, A., Koppel, J., \& Hirst, W. (2007). Silence is not golden: A case for socially shared retrieval-induced forgetting. Psychological Science, 18, 727-733.

Divis, K. M., \& Benjamin, A. S. (2014). Retrieval speeds context fluctuation: Why semantic generation enhances later learning but hinders prior learning. Memory \& Cognition, 42, 10491062.

Estes, W. K. (1955). Statistical theory of spontaneous recovery and regression. Psychological Review, 62, 145-154.

Finlay, F., Hitch, G. J., \& Meudell, P. R. (2000). Mutual inhibition in collaborative recall: Evidence for a retrieval-based account. Journal of Experimental Psychology: Learning, Memory, and Cognition, 26, 1556-1567.

Gallistel, C. R. (2009). The importance of proving the null. Psychological Review, 116, 439-453.

Geiselman, R. E., Bjork, R. A., \& Fishman, D. (1983). Disrupted retrieval in directed forgetting: A link with posthypnotic amnesia. Journal of Experimental Psychology: General, 112, 58-72.

Golding, J. M., \& Gottlob, L. R. (2005). Recall order determines the magnitude of directed forgetting in the within-participants list method. Memory \& Cognition, 33, 588-594.

Greene, R. L. (1989). Spacing effects in memory: Evidence for a twoprocess account. Journal of Experimental Psychology: Learning, Memory, and Cognition, 15, 371-377.

Harris, C. B., Barnier, A. J., \& Sutton, J. (2013). Shared encoding and the costs and benefits of collaborative recall. Journal of Experimental Psychology: Learning, Memory, and Cognition, 39, 183-195.

Harris, C. B., Keil, P. G., Sutton, J., Barnier, A. J., \& McIlwain, D. J. F. (2011). We remember, we forget: Collaborative remembering in older couples. Discourse Processes, 48, 267-303.

Hinsz, V. B., Tindale, R. S., \& Vollrath, D. A. (1997). The emerging conceptualization of groups as information processors. Psychological Bulletin, 121, 43-64.

Hirst, W., \& Echterhoff, G. (2012). Remembering in conversations: The social sharing and reshaping of memories. Annual Review of Psychology, 63, 55-79.

Howard, M. W., \& Kahana, M. J. (2002). A distributed representation of temporal context. Journal of Mathematical Psychology, 46, 269-299.

Jang, Y., \& Huber, D. E. (2008). Context retrieval and context change in free recall: Recalling from long-term memory drives list isolation. Journal of Experimental Psychology: Learning, Memory, and Cognition, 34, 112-127.

Jonker, T. R., Seli, P., \& MacLeod, C. M. (2013). Putting retrievalinduced forgetting in context: An inhibition-free, context-based account. Psychological Review, 120, 852-872.

Marion, S. B., \& Thorley, C. (2016). A meta-analytic review of collaborative inhibition and postcollaborative memory: Testing the predictions of the retrieval strategy disruption hypothesis. Psychological Bulletin, 142, 1141-1164.

Masson, M. E. J. (2011). A tutorial on a practical Bayesian alternative to null-hypothesis significance testing. Behavior Research Methods, 43, 679-690.

McGaugh, J. L. (2000). Memory - a century of consolidation. Science, $287,248-251$

Meade, M. L., Nokes, T. J., \& Morrow, D. G. (2009). Expertise promotes facilitation on a collaborative memory task. Memory, 17, $39-48$.
Mensink, G., \& Raaijmakers, J. G. W. (1988). A model for interference and forgetting. Psychological Review, 95, 434-455.

Meudell, P. R., Hitch, G. J., \& Boyle, M. M. (1995). Collaboration in recall: Do pairs of people cross-cue each other to produce new memories? The Quarterly Journal of Experimental Psychology Section A: Human Experimental Psychology, 48, 141-152.

Meudell, P. R., Hitch, G. J., \& Kirby, P. (1992). Are two heads better than one? Experimental investigations of the social facilitation of memory. Applied Cognitive Psychology, 6, 525-543.

Müller, G. E., \& Pilzecker, A. (1900). Experimentelle beiträge zur Lehre vom gedächtnis. Zeitschrift für Psychologie Ergänzungsband, 1, 1-300.

Pastötter, B., \& Bäuml, K.-H. (2007). The crucial role of postcue encoding in directed forgetting and context-dependent forgetting. Journal of Experimental Psychology: Learning, Memory, and Cognition, 33, 977-982.

Pastötter, B., \& Bäuml, K.-H. (2010). Amount of postcue encoding predicts amount of directed forgetting. Journal of Experimental Psychology: Learning, Memory, and Cognition, 36, 54-65.

Pastötter, B., Kliegl, O., \& Bäuml, K.-H. T. (2012). List-method directed forgetting: The forget cue improves both encoding and retrieval of postcue information. Memory \& Cognition, 40, 861873.

Pereira-Pasarin, L. M., \& Rajaram, S. (2011). Study repetition and divided attention: Effects of encoding manipulations on collaborative inhibition in group recall. Memory \& Cognition, 39, 968-976.

Raaijmakers, J. G. W., \& Shiffrin, R. M. (1981). Search of associative memory. Psychological Review, 88, 93-134.

Raftery, A. E. (1995). Bayesian model selection in social research. In Marsden, P. V. (Ed.), Sociological methodology 1995 (pp. 111196). Cambridge: Blackwell.

Rajaram, S. (2011). Collaboration both hurts and helps memory: A cognitive perspective. Current Directions in Psychological Science, 20, 76-81.

Rajaram, S., \& Maswood, R. (in press). Collaborative memory: A selective review of data and theory. In Byrne, J. H. (Ed.), Learning and memory: A comprehensive reference, 2nd Edn. Oxford: Academic Press.

Rajaram, S., \& Pereira-Pasarin, L. P. (2010). Collaborative memory: Cognitive research and theory. Perspectives on Psychological Science, 5, 649-663.

Roediger, IIIH. L. (1974). Inhibiting effects of recall. Memory \& Cognition, 2, 261-269.

Sahakyan, L., \& Kelley, C. M. (2002). A contextual change account of the directed forgetting effect. Journal of Experimental Psychology: Learning, Memory, and Cognition, 28, 1064-1072.

Sjolund, L. A., Erdman, M., \& Kelly, J. W. (2014). Collaborative inhibition in spatial memory retrieval. Memory \& Cognition, 42, 876-885.

Takahashi, M., \& Saito, S. (2004). Does test delay eliminate collaborative inhibition? Memory, 12, 722-731.

Thios, S. J., \& D'Agostino, P. R. (1976). Effects of repetition as a function of study-phase retrieval. Journal of Verbal Learning and Verbal Behavior, 15, 529-536.

Van Overschelde, J. P., Rawson, K. A., \& Dunlosky, J. (2004). Category norms: An updated and expanded version of the Battig and Montague (1969) norms. Journal of Memory and Language, 50, 289-335.

Wagenmakers, E.-J. (2007). A practical solution to the pervasive problems of $p$ values. Psychonomic Bulletin \& Review, 14, 779-804.

Weldon, M. S. (2000). Remembering as a social process. In Medin, D. L. (Ed.), The psychology of learning and motivation (Vol. 40, pp. 67-120) San Diego: Academic Press.

Weldon, M. S., \& Bellinger, K. D. (1997). Collective memory: Collaborative and individual processes in remembering. Journal of 
Experimental Psychology: Learning, Memory, and Cognition, 23, 1160-1175.

Weldon, M. S., Blair, C., \& Huebsch, P. D. (2000). Group remembering: Does social loafing underlie collaborative inhibition? Journal of Experimental Psychology: Learning, Memory, and Cognition, $26,1568-1577$.
Wessel, I., Zandstra, A. R., Hengeveld, H. M., \& Moulds, M. L. (2015). Collaborative recall of details of an emotional film. Memory, 23, 437-444.

Zellner, M., \& Bäuml, K.-H. (2006). Inhibitory deficits in older adults - list-method directed forgetting revisited. Journal of Experimental Psychology: Learning, Memory, and Cognition, 32, 290-300. 